This is a post-peer-review, pre-copyedit version of an article published in Metabolic Brain Disease. The final authenticated version is available online at: http://dx.doi.org/10.1007/s11011-016-9858-2

\title{
GLYCINE AND HYPERAMMONEMIA: POTENTIAL TARGET FOR THE TREATMENT OF HEPATIC ENCEPHALOPATHY
}

\section{Rune Gangsøy Kristiansen ${ }^{1,2}$, Christopher F. Rose ${ }^{3}$ and Lars Marius Ytrebø ${ }^{1}$}

\section{AfFiliations}

1. Department of Anesthesiology, Anesthesia and Critical Care Research Group, University Hospital of North Norway and UiT-The Arctic University of Norway, Troms $\emptyset$, Norway

2. Department of Anesthesiology, Ålesund Hospital, Helse Møre og Romsdal, 6010, Ålesund, Norway

3. Hepato-Neuro Laboratory, CRCHUM, Université de Montréal, Montréal, QC, Canada

KEYWORDS

Ammonia; Glutamine; Glycine; L-ornithine-phenylacetate (OP); Phenylacetylglycine

\section{Abbreviations}

$H E$ :Hepatic encephalopathy ; GCS:Glycine cleavage system; OP: L-ornithine Phenylacetate; GS:; Glutamine synthetase; $G D H$ :Glutamate dehydrogenase; $A L F$ :Acute liver failure; $N M D A$ receptor:N-methyl-D-aspartate; $N K H$ :Non-ketotic hyperglycinemia

\section{ABSTraCT}

Hepatic encephalopathy (HE) is a neuropsychiatric disorder caused by hepatic dysfunction. Numerous studies dictate that ammonia plays an important role in the pathogenesis of HE, and hyperammonemia can lead to alterations in amino acid homeostasis. Glutamine and glycine are both ammoniagenic amino acids that are increased in liver failure. Modulating the levels of glutamine and glycine has shown to reduce ammonia concentration in hyperammonemia. Ornithine Phenylacetate (OP) has consistently been shown to reduce arterial ammonia levels in liver failure by modulating glutamine levels. In addition to this, OP has also been found to modulate glycine concentration providing an additional ammonia removing effect. Data support that glycine also serves an important role in Nmethyl D-aspartate (NMDA) receptor mediated neurotransmission in HE. This potential important role for glycine in the pathogenesis of HE merits further investigations. 
This is a post-peer-review, pre-copyedit version of an article published in Metabolic Brain Disease. The final authenticated version is available online at: http://dx.doi.org/10.1007/s11011-016-9858-2

\section{HEPATIC ENCEPHALOPATHY}

Hepatic encephalopathy (HE) is a neuropsychiatric disorder caused by hepatic failure. It covers a wide spectrum of symptoms ranging from mild cognitive deficits to coma (Vilstrup et al. 2014). Liver failure leads to impairment of the urea cycle which consequently affects nitrogen handling and hyperammonemia develops. Numerous studies in animal models and patients dictate that ammonia plays an important role in the pathogenesis of HE (Clemmesen et al. 1999; Ong et al. 2003; Bernal et al. 2007). Therefore, developing treatments for reducing blood ammonia levels in patients suffering from liver disease remains a primary therapeutic strategy in HE (Rose 2012).

Hyperammonemia leads to alterations in amino acid homeostasis. Glutamine and glycine are of particular interest in this context as they have been shown to be significantly increased in blood in both humans and animal models of liver failure (Record et al. 1976; Clemmesen et al. 2000; Strauss et al. 2001). Furthermore, altered amino acid balance has been found in the brain and considered to be implicated in the pathogenesis of HE (Swain et al. 1992; Mans et al. 1994) possibly impairing neurotransmission and brain function (Swain et al. 1992).

\section{GLUTAMINE}

Glutamine is the most abundant free amino acid in the body. Synthesis of glutamine (through the amidation of glutamate) by glutamine synthetase (GS) has been targeted as an important alternative pathway in clearing ammonia, particularly in the muscle (Desjardins et al. 1999; Rose et al. 1999).

GS is also expressed in the brain, specifically in astrocytes. Under physiological conditions, GS plays an important role in the glutamate-glutamine shuttle as glutamine generated via GS in the astrocyte is shuttled to the neuron where it is converted to glutamate through glutaminase, replenishing the glutamate neurotransmitter pool in the neuron. Released glutamate from the neuron is then taken up by the astrocytes and used to subsequently generate glutamine (Martinez-Hernandez et al. 1977; Schousboe et al. 1977; Suarez et al. 2002).

During hyperammonemia with subsequent elevated levels of ammonia in the brain, GS becomes the sole ammonia removing pathway and it is believed that glutamine accumulation within the astrocytes leads to astrocyte swelling and consequently brain edema (Butterworth 2014). Furthermore, elevated glutamine levels can interfere with glutamatergic neurotransmission via the N-methyl-D-aspartate (NMDA) receptor, contributing to the development of HE (Ott and Vilstrup 2014).

During liver disease, GS expression and activity in skeletal muscles are upregulated and therefore has become a potential target for lowering blood ammonia levels (Desjardins et al. 1999). L-Ornithine LAspartate has been shown to be effective in lowering blood ammonia, possibly by increasing glutamine production via GS in muscle (Rose et al. 1999). However, concerns have been raised regarding a potential ammonia rebound effect as glutamine can be metabolized via glutaminase (located in intestines and kidneys) and thus regenerate ammonia. Therefore, glutaminase activity (ammonia generation) limits maximum ammonia removal potential via GS (Jalan and Lee 2009). 
This is a post-peer-review, pre-copyedit version of an article published in Metabolic Brain Disease. The final authenticated version is available online at: http://dx.doi.org/10.1007/s11011-016-9858-2

Studies have also shown a beneficial effect on lowering blood ammonia and HE events by administering the glutamine scavenger phenylacetate (as glycerol phenylbutyrate, pro-drug for phenylacetate) (McGuire et al. 2010; Rockey et al. 2014).

However, stimulating GS as well as removing the newly synthesized glutamine has been proposed as an efficient novel therapeutic ammonia-lowering strategy. Jalan et al. recently proposed Ornithine Phenylacetate (OP) as a novel concept to maximize ammonia clearance during liver disease. This involves stimulating ammonia removal via GS with ornithine in skeletal muscle and chelating glutamine with phenylacetate to form phenylacetylglutamine which is easily excreted in the urine (Jalan et al. 2007).

\section{GLYCINE}

Glycine is a conditionally essential, one carbon amino acid. It can be synthesized from serine, threonine and choline, and via transamination with alanine from glyoxylate (Wang et al. 2013). Glycine is metabolized by the glycine cleavage system (GCS) and glycine oxidase (Fig. 1).

GCS is a multi enzyme complex and found to be active in liver, kidney, brain and testis. In the brain GCS is confined to astrocytes (Sato et al. 1991).

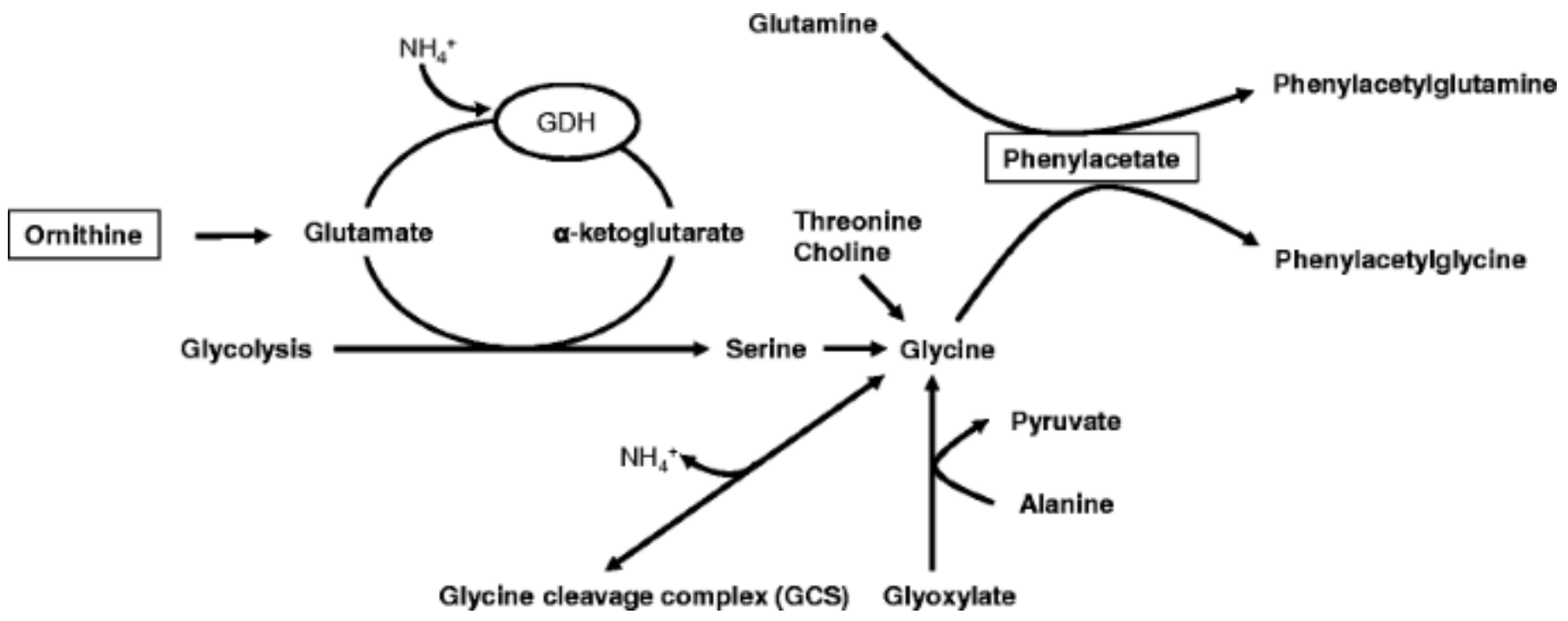

Fig. 1 The glycine pathways in relation to Ornithine Phenylacetate. Glycine can be synthesized from serine, threonine and choline, and via transamination with alanine from glyoxylate. The main catabolic pathway is the glycine cleavage system (GCS). Hyperammonemia modulates GDH reaction in favor of glutamate production. Glutamate acts as amino group donor in the synthesis of serine, driving glutamate production from $\alpha$-ketoglutarate removing ammonia. Serine can further be metabolized to glycine. GDH; glutamate dehydrogenase reaction 
This is a post-peer-review, pre-copyedit version of an article published in Metabolic Brain Disease. The final authenticated version is available online at: http://dx.doi.org/10.1007/s11011-016-9858-2

\section{GLYCINE AND AMMONIA}

Like glutamine, glycine can also be an active ammoniagenic amino acid leading to changes in both plasma and brain ammonia levels. Rudman et al. studied the ammonia liberating potential of different amino acids in patients with liver failure, and glycine was found to be important in liberating ammonia (Rudman et al. 1973). In accordance with this, we found that cirrhotic rats injected with glycine intraperitoneally (IP) leads to an increase in blood ammonia (Rose, unpublished data). Rudman et al. concluded that ammonia was released through the glycine oxidase reaction directly deaminating glycine (Kikuchi 1973; Rudman et al. 1973). However, the main catabolic pathway for glycine is considered to be through the GCS, liberating ammonia (Van Hove et al. 2005).

Studies have shown an ammonia modulating effect by the administration of the glycine scavenger sodium benzoate to liver failure patients (Sushma et al. 1992; Misel et al. 2013). Sushma et al. found that administration of benzoate to patients with cirrhotic liver failure attenuated ammonia levels, and outcome was found to be as good as with lactulose treatment (Sushma et al. 1992). Furthermore, combined treatment with benzoate and phenylacetate reduces ammonia levels, mortality and morbidity in children with urea cycle disorders (Enns et al. 2007), and has shown beneficial effects in patients with portal systemic encephalopathy (Mendenhall et al. 1986).

\section{GLYCINE AND THE NMDA-RECEPTOR}

Glycine is implicated in excitatory neurotransmission as a positive modulator on the NMDA-receptor in the cerebral cortex, cerebellum and hippocampus (Sakata et al. 2001). The NMDA-receptor is located on the postsynaptic neuronal membrane and is part of the glutamatergic neurotransmission system (Vaquero and Butterworth 2006).

Inherited defects in the GCS, known as non-ketotic hyperglycinemia (NKH), results in increased glycine in the cerebrospinal fluid leading to encephalopathy, possibly as a result of increased activation of NMDA receptors in the frontal cortex (Tada and Kure 1993). The role of ammonia in NKH has not been evaluated. Reducing glycine level in plasma and CSF with benzoate, combined with the NMDAantagonist dextromethorphan was found to improve outcome for some patients with NKH (Hamosh et al. 1998).

In the setting of liver failure, evidence has emerged suggesting that alterations in the glutamate system are involved in the pathogenesis of HE (Butterworth 1997). An increased extracellular concentration of glutamate, possibly due to decreased trafficking of glutamate between astrocytes and neurons, has been observed in ALF animals with a positive correlation with severity of $\mathrm{HE}$ and arterial ammonia levels (Michalak et al. 1996). Increased glutamate can lead to increased NMDA receptor activity. In support for this, studies have shown that inhibitors of NMDA-receptors (memantine) can improve the clinical grade of HE in animals with ALF (Vogels et al. 1997).

In a porcine model of acute liver failure (ALF) we found significantly increased levels of both arterial and brain glycine compared to sham operated animals (Kristiansen et al. 2014). Increased extracellular level of glycine in the brain has also been found in several other animal models of liver failure (Bosman et al. 1992; Swain et al. 1992; Michalak et al. 1996). Zwingmann et al. found that increased 
This is a post-peer-review, pre-copyedit version of an article published in Metabolic Brain Disease. The final authenticated version is available online at: http://dx.doi.org/10.1007/s11011-016-9858-2

brain extracellular glycine was a result of decreased uptake by Glyt-1 transporters in the brain of rats with ALF (Zwingmann et al. 2002). A potential role for glycine as a positive modulator of the NMDAreceptor in $\mathrm{HE}$ merits further investigations.

\section{ORNITHINE PHENYLACETATE; MAXIMIZING AMMONIA REMOVAL?}

During liver disease, it has been shown that increasing the synthesis of glutamine (via GS), leads to a reduction in blood ammonia (Rose et al. 1999). Furthermore, removing glutamine and glycine by the administration of phenylacetate and benzoate respectively, has been shown to reduce ammonia levels in patients with urea cycle disorders (Enns et al. 2007) and patients with HE (Rockey et al. 2014).

Ornithine Phenylacetate (OP) targets both glutamine production and removal. Ornithine increases the provision of glutamate in order to stimulate GS and generate glutamine and remove ammonia. Phenylacetate conjugates with the newly formed glutamine to form phenylacetylglutamine which cannot be metabolized by glutaminase and thus prevents an ammonia rebound effect (Jalan et al. 2007). OP has consistently been shown to decrease arterial ammonia levels both in humans and in several animal models of both acute and chronic liver failure (Ytrebo et al. 2009; Davies et al. 2009; Balasubramaniyan et al. 2012; Oria et al. 2012; Wright et al. 2012; Dadsetan et al. 2013; Ventura-Cots et al. 2013; Jover-Cobos et al. 2014). We have previously shown a significant reduction in arterial ammonia by OP treatment in a porcine model of ALF (Ytrebo et al. 2009).

However, in numerous studies (including ours) the excretion of PAGN did not correlate with the decrease in arterial ammonia. Furthermore, a study in cirrhotic patients suffering from upper gastrointestinal bleed found a recovery rate of phenylacetate as PAGN of only $31.9 \%$ over 5 days (Ventura-Cots et al. 2013). Two studies of bile duct ligated (BDL) rats reported a lack of correlation between ammonia reduction and PAGN excretion explained by kinetics of PAGN excretion and additional pathways for PAGN metabolism in animal models compared to humans (Davies et al. 2009; Dadsetan et al. 2013; Jover-Cobos et al. 2013). This led us to investigate whether alternative pathways could be involved, and interestingly enough we were able to show a significant correlation between arterial ammonia and glycine levels (Kristiansen et al. 2014).

This increase in glycine and removal of ammonia might have been mediated through the glutamate dehydrogenase (GDH) (Brusilow et al. 1980) reaction in the kidneys. Hyperammonemia modulates GDH reaction in favor of glutamate production (Cooper and Plum 1987). Glutamate acts as amino group donor in the synthesis of serine, driving glutamate production from $\alpha$-ketoglutarate removing ammonia. Serine can further be metabolized to glycine. However, glycine can also be synthesized from glyoxylate, using alanine as amino group donor, or possibly through the reversible glycine cleavage system (Kristiansen et al. 2014) (Fig. 1).

Interestingly, using a porcine model of ALF we found a positive correlation between brain glycine and intracranial pressure (Kristiansen et al. 2014). However, the mechanism for this observation remains to be studied.

Benzoate and phenylacetate conjugates with glycine and glutamine respectively, and are excreted as hippuric acid and phenylacetylglutamine. This leads to a net ammonia removal in urine and prevents 
This is a post-peer-review, pre-copyedit version of an article published in Metabolic Brain Disease. The final authenticated version is available online at: http://dx.doi.org/10.1007/s11011-016-9858-2

glutamine and glycine from being re-metabolized to ammonia. Furthermore, the replenishment of glycine and glutamine stores can remove ammonia through GS and GDH reactions (Kristiansen et al. 2014).

In our study we found phenylacetate to conjugate with glutamine as well as glycine as a highly significant increase in excretion of phenylacetylglycine was found in the OP treated animals compared to placebo treated animals (Kristiansen et al. 2014). This was accompanied by a significant attenuation of glycine which correlated with the attenuation of ammonia. Moreover, our data suggest that the conjugation between glycine and phenylacetate takes place in the kidneys.

In conclusion, glycine is an ammoniagenic amino acid as well as a positive modulator of NMDA receptors in the brain. Lowering glycine levels has demonstrated to be beneficial in lowering ammonia and well as resolving encephalopathy. Therefore, targeting glycine for the treatment of HE merits to be further investigated.

\section{REFERENCES}

1. Balasubramaniyan V, Wright $\mathrm{G}$, et al. (2012) Ammonia reduction with ornithine phenylacetate restores brain eNOS activity via the DDAH-ADMA pathway in bile duct-ligated cirrhotic rats. Am J Physiol Gastrointest Liver Physiol 302(1):G145-G152

2. Bernal W, Hall C, et al. (2007) Arterial ammonia and clinical risk factors for encephalopathy and intracranial hypertension in acute liver failure. Hepatology 46(6):1844-1852

3. Bosman DK, Deutz NE, et al. (1992) Amino acid release from cerebral cortex in experimental acute liver failure, studied by in vivo cerebral cortex microdialysis. J Neurochem 59(2):591599

4. Brusilow S, Tinker J, et al. (1980) Amino acid acylation: a mechanism of nitrogen excretion in inborn errors of urea synthesis. Science 207(4431):659-661

5. Butterworth RF (1997) Hepatic encephalopathy and brain edema in acute hepatic failure: does glutamate play a role? Hepatology 25(4):1032-1034

6. Butterworth RF (2014) Pathophysiology of brain dysfunction in hyperammonemic syndromes: the many faces of glutamine. Mol Genet Metab 113(1-2):113-117

7. Clemmesen JO, Larsen FS, Kondrup J, Hansen BA, Ott P (1999) Cerebral herniation in patients with acute liver failure is correlated with arterial ammonia concentration. Hepatology 29(3):648-653

8. Clemmesen JO, Kondrup J, et al. (2000) Splanchnic and leg exchange of amino acids and ammonia in acute liver failure. Gastroenterology 118(6):1131-1139

9. Cooper AJ, Plum F (1987) Biochemistry and physiology of brain ammonia. Physiol Rev 67(2):440-519

10. Dadsetan S, Sorensen M, et al. (2013) Interorgan metabolism of ornithine phenylacetate (OP)--a novel strategy for treatment of hyperammonemia. Biochem Pharmacol 85(1):115-123

11. Davies NA, Wright G, et al. (2009) L-ornithine and phenylacetate synergistically produce sustained reduction in ammonia and brain water in cirrhotic rats. Hepatology 50(1):155-164

12. Desjardins P, Rao KV, et al. (1999) Effect of portacaval anastomosis on glutamine synthetase protein and gene expression in brain, liver and skeletal muscle. Metab Brain Dis 14(4):273-280 
This is a post-peer-review, pre-copyedit version of an article published in Metabolic Brain Disease. The final authenticated version is available online at: http://dx.doi.org/10.1007/s11011-016-9858-2

13. Enns GM, Berry SA, et al. (2007) Survival after treatment with phenylacetate and benzoate for urea-cycle disorders. N Engl J Med 356(22):2282-2292

14. Hamosh A, Maher JF, et al. (1998) Long-term use of high-dose benzoate and dextromethorphan for the treatment of nonketotic hyperglycinemia. J Pediatr 132(4):709-713

15. Jalan R, Lee WM (2009) Treatment of hyperammonemia in liver failure: a tale of two enzymes. Gastroenterology 136(7):2048-2051

16. Jalan R, Wright G, et al. (2007) L-ornithine phenylacetate (OP): a novel treatment for hyperammonemia and hepatic encephalopathy. Med Hypotheses 69(5):1064-1069

17. Jover-Cobos M, Noiret L, et al. (2013) Ornithine phenylacetate revisited. Metab Brain Dis 28(2):327-331

18. Jover-Cobos M, Noiret L, et al. (2014) Ornithine phenylacetate targets alterations in the expression and activity of glutamine synthase and glutaminase to reduce ammonia levels in bile duct ligated rats. J Hepatol 60(3):545-553

19. Kikuchi G (1973) The glycine cleavage system: composition, reaction mechanism, and physiological significance. Mol Cell Biochem 1(2):169-187

20. Kristiansen RG, Rose CF, et al. (2014) L-ornithine phenylacetate reduces ammonia in pigs with acute liver failure through phenylacetylglycine formation: a novel ammonia-lowering pathway. Am J Physiol Gastrointest Liver Physiol 307(10):G1024-G1031

21. Mans AM, DeJoseph MR, et al. (1994) Metabolic abnormalities and grade of encephalopathy in acute hepatic failure. J Neurochem 63(5):1829-1838

22. Martinez-Hernandez A, Bell KP, Norenberg MD (1977) Glutamine synthetase: glial localization in brain. Science 195(4284):1356-1358

23. McGuire BM, Zupanets IA, Lowe ME, Xiao X, Syplyviy VA, Monteleone J, Gargosky S, Dickinson K, Martinez A, Mokhtarani M, Scharschmidt BF (2010) Pharmacology and safety of glycerol phenylbutyrate in healthy adults and adults with cirrhosis. Hepatology 51(6):20772085

24. Mendenhall CL, Rouster S, et al. (1986) A new therapy for portal systemic encephalopathy. Am J Gastroenterol 81(7):540-543

25. Michalak A, Rose C, et al. (1996) Neuroactive amino acids and glutamate (NMDA) receptors in frontal cortex of rats with experimental acute liver failure. Hepatology 24(4):908-913

26. Misel ML, Gish RG, et al. (2013) Sodium benzoate for treatment of hepatic encephalopathy. Gastroenterol Hepatol (N Y) 9(4):219-227

27. Ong JP, Aggarwal A, et al. (2003) Correlation between ammonia levels and the severity of hepatic encephalopathy. Am J Med 114(3):188-193

28. Oria M, Romero-Gimenez J, et al. (2012) Ornithine phenylacetate prevents disturbances of motor-evoked potentials induced by intestinal blood in rats with portacaval anastomosis. $\mathbf{J}$ Hepatol 56(1):109-114

29. Ott P, Vilstrup H (2014) Cerebral effects of ammonia in liver disease: current hypotheses. Metab Brain Dis 29(4):901-911

30. Record CO, Buxton B, et al. (1976) Plasma and brain amino acids in fulminant hepatic failure and their relationship to hepatic encephalopathy. Eur J Clin Investig 6(5):387-394

31. Rockey DC, Vierling JM, et al. (2014) Randomized, double-blind, controlled study of glycerol phenylbutyrate in hepatic encephalopathy. Hepatology 59(3):1073-1083

32. Rose CF (2012) Ammonia-lowering strategies for the treatment of hepatic encephalopathy. Clin Pharmacol Ther 92(3):321-331 
This is a post-peer-review, pre-copyedit version of an article published in Metabolic Brain Disease. The final authenticated version is available online at: http://dx.doi.org/10.1007/s11011-016-9858-2

33. Rose C, Michalak A, et al. (1999) L-ornithine-L-aspartate lowers plasma and cerebrospinal fluid ammonia and prevents brain edema in rats with acute liver failure. Hepatology 30(3):636640

34. Rudman D, Galambos JT, et al. (1973) Comparison of the effect of various amino acids upon the blood ammonia concentration of patients with liver disease. Am J Clin Nutr 26(9):916-925

35. Sakata Y, Owada Y, et al. (2001) Structure and expression of the glycine cleavage system in rat central nervous system. Brain Res Mol Brain Res 94(1-2):119-130

36. Sato K, Yoshida S, et al. (1991) Glycine cleavage system in astrocytes. Brain Res 567(1):64-70

37. Schousboe A, Svenneby G, Hertz L (1977) Uptake and metabolism of glutamate in astrocytes cultured from dissociated mouse brain hemispheres. J Neurochem 29(6):999-1005

38. Strauss GI, Knudsen GM, et al. (2001) Cerebral metabolism of ammonia and amino acids in patients with fulminant hepatic failure. Gastroenterology 121(5):1109-1119

39. Suarez I, Bodega G, Fernandez B (2002) Glutamine synthetase in brain: effect of ammonia. Neurochem Int 41(2-3):123-142

40. Sushma S, Dasarathy S, et al. (1992) Sodium benzoate in the treatment of acute hepatic encephalopathy: a double-blind randomized trial. Hepatology 16(1):138-144

41. Swain MS, Bergeron M, et al. (1992) Monitoring of neurotransmitter amino acids by means of an indwelling cisterna magna catheter: a comparison of two rodent models of fulminant liver failure. Hepatology 16(4):1028-1035

42. Tada K, Kure S (1993) Non-ketotic hyperglycinaemia: molecular lesion, diagnosis and pathophysiology. J Inherit Metab Dis 16(4):691-703

43. Van Hove JL, Vande Kerckhove K, et al. (2005) Benzoate treatment and the glycine index in nonketotic hyperglycinaemia. J Inherit Metab Dis 28(5):651-663

44. Vaquero J, Butterworth RF (2006) The brain glutamate system in liver failure. J Neurochem 98(3):661-669

45. Ventura-Cots M, Arranz JA, et al. (2013) Safety of ornithine phenylacetate in cirrhotic decompensated patients: an open-label, dose-escalating, single-cohort study. J Clin Gastroenterol 47(10):881-887

46. Vilstrup H, Amodio P, Bajaj J, Cordoba J, Ferenci P, Mullen KD, Weissenborn K, Wong P (2014) Hepatic encephalopathy in chronic liver disease: 2014 practice guideline by the American Association for the Study of Liver Diseases and the European Association for the Study of the liver. Hepatology 60(2):715-735

47. Vogels BA, Maas MA, et al. (1997) Memantine, a noncompetitive NMDA receptor antagonist improves hyperammonemia-induced encephalopathy and acute hepatic encephalopathy in rats. Hepatology 25(4):820-827

48. Wang W, Wu Z, et al. (2013) Glycine metabolism in animals and humans: implications for nutrition and health. Amino Acids 45(3):463-477

49. Wright G, Vairappan B, et al. (2012) Reduction in hyperammonaemia by ornithine phenylacetate prevents lipopolysaccharide-induced brain edema and coma in cirrhotic rats. Liver Int 32(3):410-419

50. Ytrebo LM, Kristiansen RG, et al. (2009) L-ornithine phenylacetate attenuates increased arterial and extracellular brain ammonia and prevents intracranial hypertension in pigs with acute liver failure. Hepatology 50(1):165-174

51. Zwingmann C, Desjardins P, et al. (2002) Reduced expression of astrocytic glycine transporter (Glyt-1) in acute liver failure. Metab Brain Dis 17(4):263-273 